\section{Exogenous Application of Nitrogen and Cytokinin on Growth, Carbohydrate, and Antioxidant Metabolism of Creeping Bentgrass after De-submergence}

\author{
Qiang Liu and Yiwei Jiang ${ }^{1}$ \\ Department of Agronomy, Purdue University, West Lafayette, IN 47907
}

Additional index words. Agrostis stolonifera, flooding, foliar application, metabolism, recovery

\begin{abstract}
Recovery from submergence stress is vital for plant regrowth. The objective of this study was to characterize plant growth, carbohydrate, and antioxidant metabolism of creeping bentgrass (Agrostis stolonifera) to foliar application of nitrogen and cytokinin (CK) after de-submergence. Creeping bentgrass (cv. Penncross and 007) were submerged under the water for 14 days and then foliar-sprayed at 1, 2, 3, 7, and 14 days after desubmergence with six types of chemical treatments, respectively: 1) water (W);2) $10 \mathrm{~mm}$ urea (N10); 3) 20 mM urea (N20); 4) $10 \mu M$ CK; 5) N10 with CK (N10CK); and 6) N20 with CK (N20CK). Leaves were harvested at 20 days after chemical applications for various measurements. Compared with the nonstressed plants, plant height (HT), chlorophyll index (Chl), leaf dry weight (DW), water-soluble carbohydrate content (WSC), activities of superoxide dismutase (SOD), and ascorbate peroxidase (APX) decreased, but catalase (CAT) and peroxidase (POD) activities, malondialdehyde (MDA), and total soluble protein (TSP) content increased in both cultivars exposed to 14 days of submergence. After desubmergence, plants treated with $\mathrm{N}$ alone (N10, N20) or combined with $\mathrm{CK}$ (N10CK, N20CK) generally had higher HT, DW, Chl, TSP, and a lower amount of MDA, compared with treatments of W or CK alone, whereas treatment using CK resulted in higher WSC for both cultivars. Foliar applications of $\mathrm{N}$ and $\mathrm{CK}$ had some effect on SOD, CAT, POD, and APX activities after de-submergence, but the effects were not consistent across chemicals and cultivars. The results indicated that foliar application of $\mathrm{N}$ or combined with CK promoted plant growth and reduced lipid peroxidation after de-submergence. The results also suggested a more positive role of foliar $\mathbf{N}$ application in comparison with a complex regulation of $\mathrm{CK}$ on creeping bentgrass regrowth after de-submergence.
\end{abstract}

Heavy and frequent rainfall often creates a flooding environment for plants grown in a poorly drained site. As a result, plants can be completely covered by water, which negatively affects plant growth and physiology due to lack of oxygen. Plant survival in a submerged condition depends on severity of stress, species, and genotypes, as well as survival strategies (Bailey-Serres and Voesenek, 2008; Liu and Jiang, 2015; Yu et al., 2012). The mechanisms of escape and quiescence are strategies for plant tolerance to submergence stress (Bailey-Serres and Voesenek, 2008). Although these mechanisms can be crucial for a particular plant growing under submergence stress, recoverability after de-submergence could also play an important role in plant survival (Alpuerto et al., 2016; Gautam et al., 2016; Panda et al., 2008).

On de-submergence, variations of plant growth, physiological, and molecular alterations have been observed in different species or cultivars exposed to various submerged conditions (Alpuerto et al., 2016; Luo et al., 2011; Yu et al., 2012). In alligator weed

Received for publication 13 Sept. 2016. Accepted for publication 9 Oct. 2016.

${ }^{1}$ Corresponding author. E-mail: yjiang@purdue. edu.

1602
Rapid recovery from submergence stress is a desirable trait that allows plants to more quickly resume normal function. Exogenous application of nutrients or plant growth regulators may promote plant recovery after de-submergence. Nitrogen $(\mathrm{N})$ is a major component of chlorophyll molecules, therefore, exogenous $\mathrm{N}$ supply can enhance chlorophyll biosynthesis, which then reduces submergence stress-induced chlorosis. Foliar application of $\mathrm{N}$ after de-submergence substantially increased chlorophyll and soluble sugar contents, leaf area, dry matter weight, specific leaf weight, and tiller regeneration ability of rice (Gautam et al., 2014a, 2014b). Exogenous application of $\mathrm{N}$ also positively affected the growth and physiology of the plants under waterlogging conditions. Habibzadeh et al. (2013) reported that foliar application of $\mathrm{N}$ compounds before and after stress improved growth and antioxidant activities of canola (Brassica napus). In sugarcane (Saccharum officinarum), the addition of $\mathrm{N}$ compounds increased leaf area, shoot weight and nitrate reductase activity, and decreased MDA content, compared with untreated plants exposed to waterlogging stress (Radha et al., 2015). These research findings indicated that the exogenous $\mathrm{N}$ application could alleviate the adverse effects of flooding stress.

CK regulates a wide array of processes crucial to plant growth and development and abiotic stress tolerance (O'Brien and Benkova, 2013; Zwack and Rashotte, 2015). Ren et al. (2016) demonstrated that application of CK after waterlogging delayed leaf senescence, increased chlorophyll content, and improved photosynthetic performance of summer maize (Zea mays). At the molecular level, expression of the ipt gene controlling CK biosynthesis did not result in CK accumulation in submerged plants until the stress was removed (Huynh et al., 2005). Transgenic wheat (Triticum aestivum) plants carrying the ipt gene were more tolerant of flooding than wild type plants (Tereshonok et al., 2011). CK is beneficial in improving plant growth and development, but the effects of CK application on plant growth and physiology after de-submergence are not clear, particularly in comparison with the effectiveness of $\mathrm{N}$ application.

Creeping bentgrass is a widely used coolseason turfgrass on golf greens and fairways in temperate regions. These grass plants can be subjected to submerged conditions due to high and frequent precipitation combined with poor soil drainage. Recoverability from submergence stress is an important trait that influences grass quality and performance, but physiological mechanisms of the plants to de-submergence are not known. In practice, turfgrass managers often have few strategies at hand to recover grass from prolonged submergence stress. Whether foliar applications of $\mathrm{N}$ or $\mathrm{CK}$ alone, or in combination, promotes plant growth and metabolic activities after de-submergence is not well understood in plant species, including turfgrass. Therefore, the objective of the experiment was to investigate plant growth, carbohydrate, and antioxidant metabolism of creeping bentgrass to exogenous $\mathrm{N}$ and $\mathrm{CK}$ application after 
de-submergence. The results from the study would reveal physiological mechanisms of perennial plants to recovery after submergence.

\section{Materials and Methods}

Plant materials and growing conditions. Creeping bentgrass cultivars Penncross and 007 were chosen for the experiment. Penncross is a commonly used cultivar but is sensitive to waterlogging stress (Jiang and Wang, 2006). 007 is a newly developed cultivar that shows good overall turf quality (NTEP, 2016). On 2 July 2015, the seeds were sown in plastic pots $(10 \mathrm{~cm}$ diameter, $9 \mathrm{~cm}$ deep) containing top soil in a greenhouse at Purdue University, West Lafayette, IN. Plants were watered four times a week and fertilized once a week with a soluble fertilizer (N-P-K, 24-8-16) (Scotts Inc., Marysville, $\mathrm{OH})$ to supply $\approx 290 \mathrm{~kg} \mathrm{~N} / \mathrm{ha}$ during the whole experiment. Plants were mowed three times a week at $3 \mathrm{~cm}$. During the growing periods, the average air temperatures and photosynthetic photon flux density $(P P F D)$ in the greenhouse were $20 \pm 1.5{ }^{\circ} \mathrm{C}$ and $700 \mu \mathrm{mol} \cdot \mathrm{m}^{-2} \cdot \mathrm{s}^{-1}$, respectively. The grass was cut to $3 \mathrm{~cm}$ before submergence stress.

Submergence stress. Submergence stress began on 19 Nov. and ended on 3 Dec. 2015. The submerged pots were placed into plastic containers $(60 \mathrm{~cm}$ length $\times 43 \mathrm{~cm}$ width $\times 31 \mathrm{~cm}$ height) and tap water ( $\mathrm{pH}$ of 6.5 , electrical conductivity of $1.2 \mathrm{dS} \cdot \mathrm{m}^{-1}$ ) was added to $10 \mathrm{~cm}$ above the grass canopy at the beginning of the treatment. During the periods of stress, the average air temperatures and PPFD were the same as the conditions indicated above. The average water temperature was $19.0 \pm 1.0{ }^{\circ} \mathrm{C}$. The nonsubmerged control pots were placed in the same size containers, but without the excess water, and irrigated every $2 \mathrm{~d}$. No nutrients were supplied to the plants during the period of submergence stress. Algae were removed if they accumulated. On 3 Dec. 2015 (after 14 d of stress), all submerged plants were removed from the containers, and leaf samplings were made from randomly selected pots for various measurements for both the control and submerged plants, and the remaining pots were allowed for recovery.

Application of nitrogen and $C K$ after de-submergence. The control and submerged plants were foliar-sprayed at 1, 2, 3, 7, and $14 \mathrm{~d}$ after de-submergence with the following treatments, respectively: 1) water (W); 2) $10 \mathrm{~mm}$ urea (N10); 3) $20 \mathrm{~mm}$ urea (N20); 4) $15 \mu \mathrm{M}$ cytokinin (transzeatin riboside, ZR) (CK); 5) $10 \mathrm{~mm}$ urea $+15 \mu \mathrm{M}$ cytokinin (N10CK); and 6) $20 \mathrm{~mm}$ urea $+15 \mu \mathrm{M}$ cytokinin (N20CK). Pots were sprayed with $10 \mathrm{~mL}$ of solutions at each date for $\mathrm{N}$ and $\mathrm{CK}$ applications as described above in this paragraph. At $20 \mathrm{~d}$ after spraying, leaves were collected for various measurements.

Measurements. Various measurements were made for both control and submerged plants as well as for the plots that received post desubmergence chemical applications. HT was recorded by measuring the length of the longest leaf blades. Chl was measured on five randomly selected leaves per pot using the
Table 1. Effects of $14 \mathrm{~d}$ submergence stress on plant height (HT), chlorophyll index (Chl), leaf dry weight (DW), content of water soluble carbohydrate (WSC), and total soluble protein (TSP) across two creeping bentgrass cultivars.

\begin{tabular}{lccccc}
\hline Treatment & HT $(\mathrm{cm})$ & Chl & DW $(\mathrm{g})$ & WSC $\left(\mathrm{mg} \cdot \mathrm{g}^{-1} \mathrm{DW}\right)$ & TSP $\left(\mathrm{mg} \cdot \mathrm{g}^{-1} \mathrm{FW}\right)$ \\
\hline Nonstress & $7.6 \pm 1.4 \mathrm{a}$ & $83.3 \pm 4.3 \mathrm{a}$ & $0.59 \pm 0.1 \mathrm{a}$ & $30.7 \pm 5.4 \mathrm{a}$ & $4.8 \pm 0.8 \mathrm{~b}$ \\
Submergence & $3.3 \pm 1.2 \mathrm{~b}$ & $40.9 \pm 4.1 \mathrm{~b}$ & $0.33 \pm 0.02 \mathrm{~b}$ & $17.3 \pm 6.7 \mathrm{~b}$ & $5.9 \pm 0.4 \mathrm{a}$ \\
\hline
\end{tabular}

Means followed by same letter are not significantly different at $P<0.05$ between nonstress and submergence

for a given measurement. Data are presented as value \pm SD.

Table 2. Effects of $14 \mathrm{~d}$ submergence stress on activities of superoxide dismutase (SOD), catalase (CAT), peroxidase (POD) and ascorbate peroxidase (APX), and malondialdehyde (MDA) content across two creeping bentgrass cultivars.

\begin{tabular}{lccccc}
\hline & & CAT & POD & APX & \\
\cline { 3 - 4 } Treatment & SOD (unit/mg protein $)$ & \multicolumn{2}{c}{$\left(\mu \mathrm{mol} \cdot \mathrm{min}^{-1} \cdot \mathrm{mg}^{-1}\right.$ protein $)$} & MDA $\left(\mathrm{mg} \cdot \mathrm{g}^{-1} \mathrm{FW}\right)$ \\
\hline Nonstress & $257.2 \pm 74.3 \mathrm{a}$ & $24.8 \pm 40.3 \mathrm{~b}$ & $0.25 \pm 0.1 \mathrm{~b}$ & $1.25 \pm 0.2 \mathrm{a}$ & $1.7 \pm 0.3 \mathrm{~b}$ \\
Submergence & $117.3 \pm 40.6 \mathrm{~b}$ & $54.1 \pm 10.1 \mathrm{a}$ & $0.41 \pm 0.1 \mathrm{a}$ & $0.41 \pm 0.2 \mathrm{~b}$ & $15.1 \pm 3.3 \mathrm{a}$ \\
\hline
\end{tabular}

Means followed by same letter are not significantly different at $P<0.05$ between nonstress and submergence for a given measurement. Data are presented as value \pm SD.
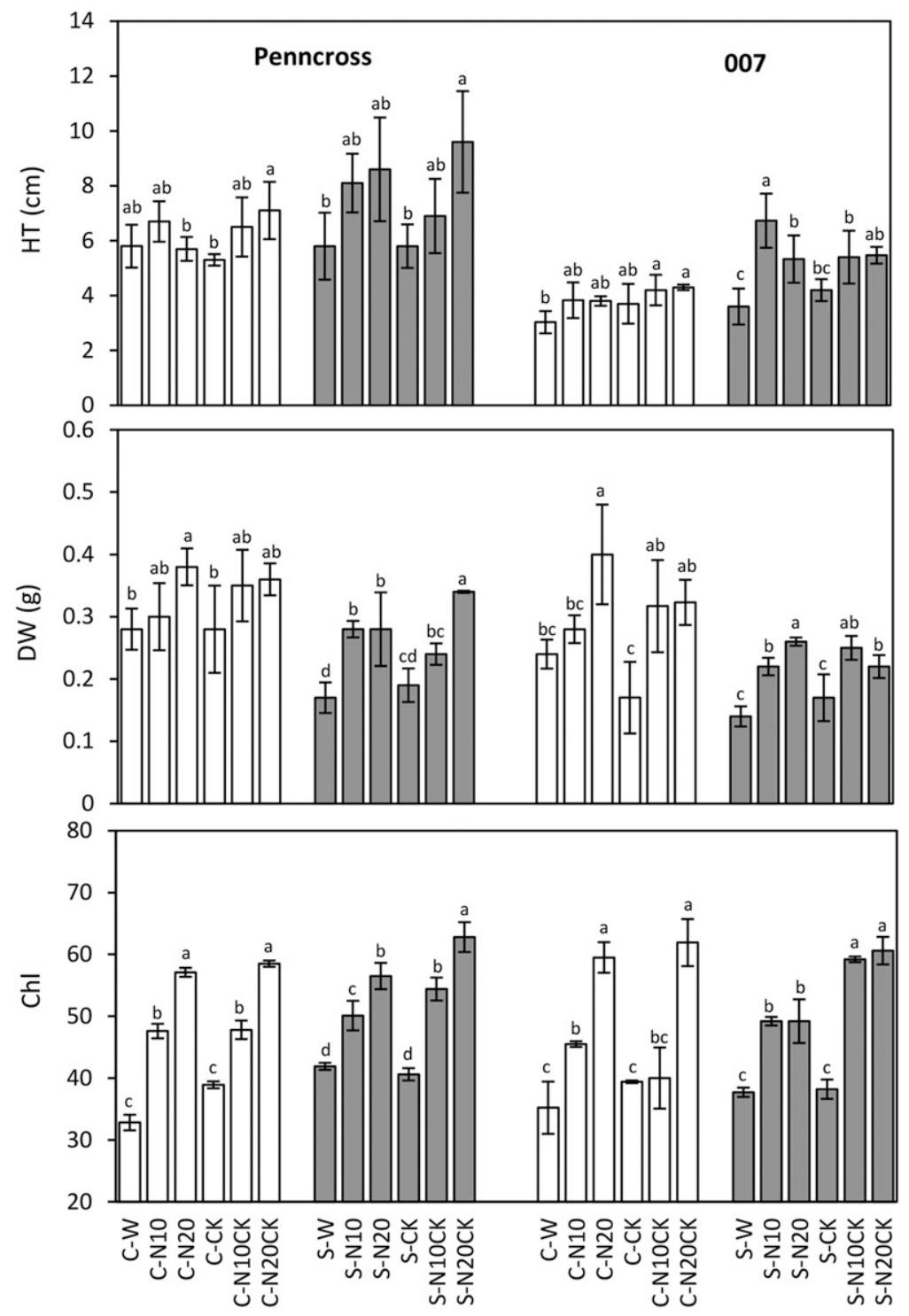

Fig. 1. Plant height (HT), leaf dry weight (DW), and chlorophyll index (Chl) of creeping bentgrass cultivars as affected by foliar application of water (W), $10 \mathrm{~mm}$ urea (N10), $20 \mathrm{~mm}$ urea (N20), $15 \mu \mathrm{M}$ cytokinin (CK), $10 \mathrm{~mm}$ urea plus $15 \mu \mathrm{m}$ cytokinin (N10CK), and $20 \mathrm{~mm}$ urea plus $15 \mu \mathrm{m}$ cytokinin (N20CK) in unstressed (C) and submerged (S) plants after $20 \mathrm{~d}$ of de-submergence and at $14 \mathrm{~d}$ of desubmergence. Means followed by same letter are not significantly different at $P<0.05$ within $\mathrm{C}$ or $\mathrm{S}$ for a given cultivar. Bars indicate SD. 
SPAD 502 Plus Chlorophyll Meter (Spectrum Technologies, Inc., IL). Leaf tissues were collected for various measurements including DW, carbohydrate, and enzyme activity. The total WSC content was determined using the anthrone method (Koehler, 1952) with some modifications (Yu et al., 2012). Briefly, WSC was extracted from $50 \mathrm{mg}$ of ground leaf tissue with $1 \mathrm{~mL}$ double-distilled water. The extract was shaken for $10 \mathrm{~min}$ and centrifuged at $11,000 g_{\mathrm{n}}$ for $10 \mathrm{~min}$, and the supernatant was collected. The extraction was repeated three times for all samples and the supernatants from each extraction were pooled for each sample. A $1 \mathrm{~mL}$ aliquot of extract was mixed with $7 \mathrm{~mL}$ freshly prepared anthrone [200 $\mathrm{mg}$ anthrone $\left.+100 \mathrm{~mL} 72 \%(\mathrm{w} / \mathrm{w}) \mathrm{H}_{2} \mathrm{SO}_{4}\right]$ and placed in a boiling water bath for $8 \mathrm{~min}$. After cooling, absorbance at $625 \mathrm{~nm}$ was read. The standard curve was determined using glucose in a range of 5 to $250 \mu \mathrm{g} \cdot \mathrm{mL}^{-1}$.

To extract the soluble protein, leaf tissues were frozen using liquid nitrogen, and ground into a fine powder. About $50 \mathrm{mg}$ powder was mixed with $1 \mathrm{~mL}$ of extraction buffer $(50 \mathrm{~mm}$ potassium phosphate, $1 \mathrm{~mm}$ ethylenediaminetetraacetic acid, $1 \%$ polyvinylpyrrolidone, $\mathrm{pH}$ 7.8). The mixture was centrifuged at $15,000 g_{n}$ for $30 \mathrm{~min}$ at $4{ }^{\circ} \mathrm{C}$, and the supernatant was collected for enzyme assay. The TSP content was measured using the method of Bradford (1976). The activities of SOD, CAT, POD, and APX were assayed by using the methods of Zhang and Kirkham (1996) with minor modifications (Liu and Jiang, 2015).

Lipid peroxidation was measured in terms of MDA content (Dhindsa et al., 1981) with some modifications (Liu and Jiang, 2015). A $0.5 \mathrm{~mL}$ aliquot of supernatant was mixed with $2 \mathrm{~mL}$ of $20 \%$ trichloroacetic acid containing $0.5 \%$ thiobarbituric acid. The mixture was heated at $95{ }^{\circ} \mathrm{C}$ for 30 min, quickly cooled, and then centrifuged at $10,000 \mathrm{~g}_{\mathrm{n}}$ for $10 \mathrm{~min}$. The absorbance was read at 532 and $600 \mathrm{~nm}$ (Heath and Packer, 1968). MDA content was calculated using an extinction coefficient of $155 \mathrm{~mm}^{-1} \cdot \mathrm{cm}^{-1}$.

Experimental design and statistical analyses. The experiment was a split-split-plot design with three replications. The submergence treatments were as the whole-plot, chemical treatments as the subplot, and the cultivars as the sub-subplot. The pots were randomly assigned within each treatment. The analysis of variance (ANOVA) and least significant difference mean separation tests were performed using SAS Procedures Guide (2004).

\section{Results and Discussion}

Submergence and cultivar effects before de-submergence. ANOVA indicated significant submergence effects on all traits and cultivar effects on HT and WSC, but no submergence by cultivar interactions were observed for all traits except for APX (data not shown). Across cultivars, HT, Chl, DW, WSC, SOD, and APX activities significantly decreased by $52.4 \%, 50.9 \%, 44.1 \%, 43.6 \%, 54.4 \%$, and $67.2 \%$, respectively; whereas TSP, CAT, and POD activities, and MDA content increased by
$18.0 \%, 1.2$-fold, $96.0 \%$, and 8.1 -fold at $14 \mathrm{~d}$ of stress, respectively (Tables 1 and 2).

Other studies have shown that submergence either decreases or enhances plant growth, depending on the type of species and survival strategy (Bailey-Serres and Voesenek, 2008). Creeping bentgrass is tolerant to low cutting, which could allow the plants to conserve energy to increase survival under a short-term submergence stress. However, severe reductions in HT, DW, Chl, and WSC in Penncross and 007 indicated that physiological damages occurred to creeping bentgrass under the water. Decreased WSC of shoots and roots were also observed in bermudagrass (Cynodon dactylon) exposed to an increase of depth and duration of submergence
(Tan et al., 2010). Moreover, large natural variations of plant growth and leaf color were found in perennial ryegrass accessions under $7 \mathrm{~d}$ of submergence, demonstrating diverse survival mechanisms for this species ( $\mathrm{Yu}$ et al., 2012).

In this study, submergence stress altered the activities of antioxidant enzymes and increased MDA content, potentially leading to lipid peroxidation in creeping bentgrass (Table 2). Antioxidant enzymes play an important role in the removal of toxicity caused by an accumulation of reactive oxygen species under stress conditions. As shown in previous studies, the activities of antioxidant enzymes increased, decreased, and remained unchanged in different plant species, cultivars, and plant
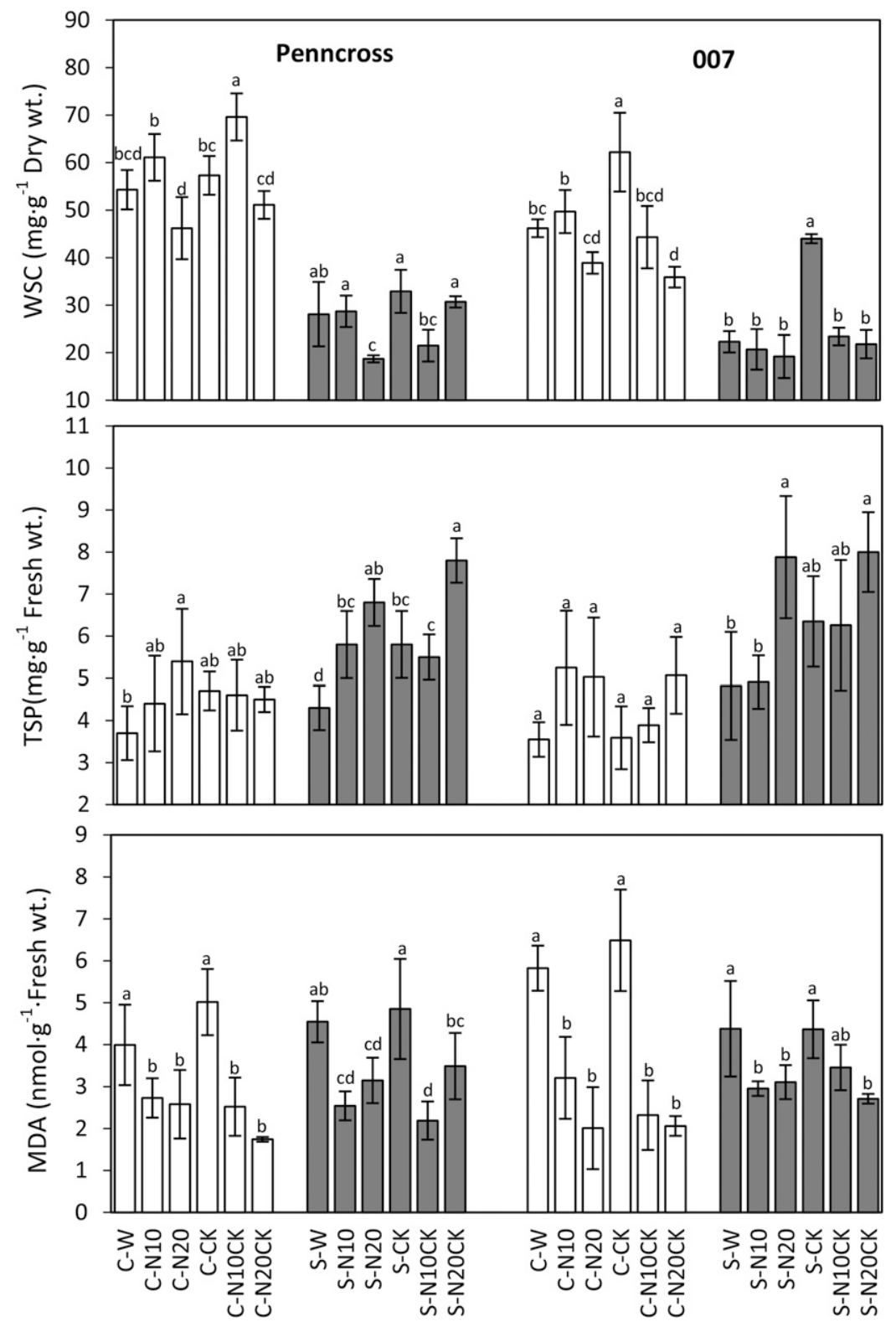

Fig. 2. Water soluble carbohydrate (WSC), total soluble protein (TSP), and malondialdehyde (MDA) contents of creeping bentgrass cultivars as affected by foliar application of water (W), $10 \mathrm{~mm}$ urea (N10), $20 \mathrm{~mm}$ urea (N20), $15 \mu \mathrm{m}$ cytokinin (CK), $10 \mathrm{~mm}$ urea plus $15 \mu \mathrm{m}$ cytokinin (N10CK), and $20 \mathrm{~mm}$ urea plus $15 \mu \mathrm{m}$ cytokinin (N20CK) in unstressed (C) and submerged (S) plants after $20 \mathrm{~d}$ of desubmergence and at $14 \mathrm{~d}$ of de-submergence. Means followed by same letter are not significantly different at $P<0.05$ within $\mathrm{C}$ or $\mathrm{S}$ for a given cultivar. Bars indicate SD. 
tissues under submergence stress (Liu and Jiang, 2015; Panda and Sarkar, 2013; Tan et al., 2010). In rice, submergence increased MDA content (Lal et al., 2015), but the tolerant cultivar had higher antioxidant enzyme activities and chlorophyll content and lower MDA content than that of the intolerant cultivars during submergence (Panda and Sarkar, 2013).

Submergence, cultivar, and chemical effects after de-submergence. ANOVA indicated significant submergence, cultivar, and chemical application effects were found for most traits except for no cultivar effects on TSP, APX, and MDA, no submergence effects on POD, and no chemical application effects on POD, CAT, and SOD (data not shown). No submergence $\times$ cultivar $\times$ chemical application interactions were identified for all traits except for Chl, WSC, and MDA (data not shown).

Nitrogen and $C K$ effects on growth and Chl after de-submergence. Different chemical applications resulted in significant differences in HT, DW, and Chl for plants previously exposed to nonstress or de-submergence conditions for both cultivars (Fig. 1). After $20 \mathrm{~d}$ of de-submergence, higher HT, DW, and Chl were generally found in plants treated with N10, N20, N10CK, or N20CK, whereas the lowest HT and DW were observed in plants treated with S-W or S-CK alone for both cultivars (Fig. 1). For example, compared with plants sprayed with S-W, Chl was $\approx 20 \%, 35 \%$, $30 \%$, and $50 \%$ higher for Penncross and $31 \%$, $31 \%, 57 \%$, and $61 \%$ higher for 007 under S-N10, S-N20, S-N10CK, and S-N20CK, respectively. The results demonstrated that separate $\mathrm{W}$ or CK treatments had no effect on the recovery of HT, DW, and Chl, but foliar applications of $\mathrm{N}$ alone or in combination with $\mathrm{CK}$ promoted plant growth and maintained leaf color. In a previous study, the positive effects of foliar $\mathrm{N}$ application were also noted in rice plants exposed to de-submergence (Gautam et al., 2014a, 2014b).

Nitrogen and CK effects on WSC, TSP, and MDA after de-submergence. Plant WSC varied with chemical applications under both nonstress and de-submergence conditions (Fig. 2). On recovery, WSC was not consistent between S-N10 and S-N10CK and between S-N20 and S-N20CK in Penncross, but $\mathrm{CK}$ alone led to higher values of WSC along with S-N10 and S-N20CK. For 007, WSC was significantly higher under S-CK treatment than all other chemical applications at de-submergence. The $\mathrm{N}$ application promoted recovery of WSC in rice (Gautam et al., 2014a, 2014b), but our results showed that CK was more effective on WSC than $\mathrm{N}$, at least for 007. Higher $\mathrm{Chl}$ found in S-N10CK and S-N20CK than in S-N10 and S-N20, along with positive CK effects on WSC as opposed to growth traits such HT and DW suggested a complex regulation of CK to the recovery of regrowth and physiology of creeping bentgrass. In maize (Z. mays), application of CK after dewaterlogging delayed leaf senescence, increased chlorophyll content, and improved photosynthetic performance (Ren et al., 2016). It seemed that CK effects on plant recovery largely depended on stress intensity and type of measurements after flooding stress.

Variations of TSP were found among various chemical applications for plants previously exposed to nonstress and de-submergence conditions, with the exception of 007 at recovery (Fig. 2). The highest value of TSP was found with S-N20CK and the lowest with W; whereas for 007, S-N20, and S-N20CK had higher TSP than other applications. The application of higher $\mathrm{N}$ concentrations resulted in higher TSP in both cultivars. However, similar levels of TSP between S-W and S-CK, between S-N10 and S-N10CK, and between S-N20 and S-N20CK suggested that it was $\mathrm{N}$, not $\mathrm{CK}$, that accounted for the TSP recovery after submergence.

Generally, S-W or S-CK exhibited higher MDA content, whereas S-N10, S-N20, S-N10CK, or S-N20CK had lower MDA content in plants exposed to nonstress and de-submergence conditions (Fig. 2). The lowest MDA content found was under N10CK for Penncross and N20CK for 007 after desubmergence. The results indicated that $\mathrm{W}$ or CK alone did not limit the level of lipid peroxidation, but the application of $\mathrm{N}$ or $\mathrm{N}$ plus CK could alleviate oxidative stress injury
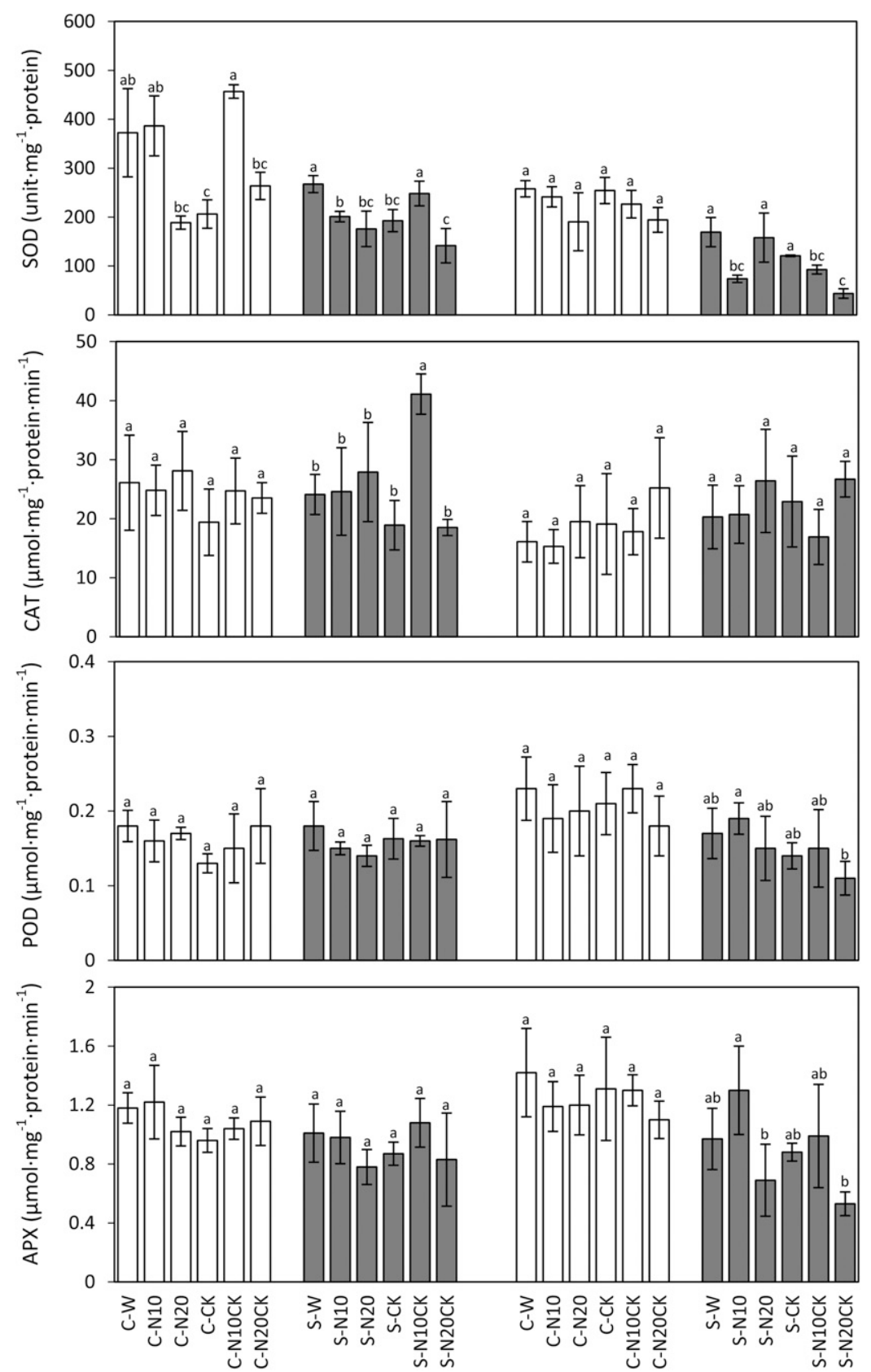

Fig. 3. Activities of superoxide dismutase (SOD), catalase (CAT), peroxidase (POD), and ascorbate peroxidase (APX) of creeping bentgrass cultivars as affected by foliar application of water (W), $10 \mathrm{~mm}$ urea (N10), $20 \mathrm{~mm}$ urea (N20), $15 \mu \mathrm{m}$ cytokinin (CK), $10 \mathrm{~mm}$ urea plus $15 \mu \mathrm{m}$ cytokinin (N10CK), and $20 \mathrm{~mm}$ urea plus $15 \mu \mathrm{m}$ cytokinin (N20CK) in unstressed (C) and submerged (S) plants after $20 \mathrm{~d}$ of desubmergence and at $14 \mathrm{~d}$ of de-submergence. Means followed by same letter are not significantly different at $P<0.05$ within $\mathrm{C}$ or $\mathrm{S}$ for a given cultivar. Bars indicate SD. 
after de-submergence. This was further supported by the higher HT, DW, and Chl levels under $\mathrm{N}$ or $\mathrm{N}$ plus $\mathrm{CK}$ than that of $\mathrm{W}$ or $\mathrm{CK}$ alone (Fig. 1). According to Gautam et al. (2014a, 2014b), an increased MDA content was also found in rice after de-submergence, but to a greater extent in the susceptible cultivar, suggesting that a potentially higher degree of oxidative injury contributed to poor tolerance of the susceptible cultivar.

Nitrogen and $C K$ effects on antioxidant enzyme activities after de-submergence. All chemical applications did not alter activities of antioxidant enzymes in both cultivars under nonstress conditions, except for higher SOD for Penncross under N10CK (Fig. 3). On de-submergence, activities of the antioxidant enzymes were not consistent in the two cultivars of creeping bentgrass across various chemical applications. Specifically, higher SOD activities were found in Penncross treated with S-W or S-N10CK with lower SOD activities under S-N20 and S-N20CK; whereas plants treated with S-W, S-N20CK, or S-CK had higher SOD activities in 007 after de-submergence. S-N10CK significantly increased CAT activities in Penncross, but CAT activities remained unchanged across applications for 007 after de-submergence. For Penncross, POD and APX activities did not vary across various applications during recovery, whereas higher POD and APX activities were found in S-N10 compared with S-N20CK in 007.

After de-submergence, a sudden increase in $\mathrm{O}_{2}$ may lead to an increased production of reactive oxygen species and simulate antioxidant enzyme activities (Blokhina et al., 2003). The relatively lower SOD activities in Penncross or the lower POD and APX activities in 007 treated with S-20NCK might indicate a lesser degree of oxidative injury for those plants after de-submergence, which was partially consistent with MDA content (Fig. 2) and contributed to plant regrowth (Fig. 1). In rice, $\mathrm{N}$ application did not cause a significate reduction of SOD and CAT activities after submergence (Gautam et al., 2014a), but the tolerant cultivar had significantly higher SOD, CAT, POD, and APX activities than the intolerant cultivars (Panda and Sarkar, 2013). It seems that the antioxidant responses of plants to $\mathrm{N}$ or $\mathrm{CK}$ application are varied and complex, depending on species, cultivars, and experimental conditions.

In summary, submergence stress significantly decreased plant growth, Chl, and WSC, altered antioxidant enzyme activities, and increased MDA content in creeping bentgrass cultivars. After de-submergence, plants treated with $\mathrm{N}$ alone or combined with $\mathrm{CK}$ generally had higher HT, DW, Chl, and TSP, and a lower amount of MDA, compared with plants treated with $\mathrm{W}$ or $\mathrm{CK}$ alone. Plants treated with $\mathrm{CK}$ alone promoted WSC. Effects of N and CK on antioxidant activities after de-submergence were not consistent. The enhanced recovery by foliar application of $\mathrm{N}$, or combined with $\mathrm{CK}$, may be accomplished mainly through promoting growth, Chl, and TSP and reducing lipid peroxidation after de-submergence.

\section{Literature Cited}

Alpuerto, J.B., R.M. Hussain, and T. Fukao. 2016. The key regulator of submergence tolerance, SUB1A, promotes photosynthetic and metabolic recovery from submergence damage in rice leaves. Plant Cell Environ. 39:672-684.

Bailey-Serres, J. and L.A.C.J. Voesenek. 2008 Flooding stress: Acclimations and genetic diversity. Annu. Rev. Plant Biol. 59:313-319.

Blokhina, O., E. Virolainen, and K.V. Fagestedt. 2003. Antioxidants, oxidative damage, and oxygen deprivation stress: A review. Ann. Bot. (Lond.) 91:179-194.

Bradford, M.M. 1976. A rapid and sensitive method for the quantitation of microgram quantities of protein utilizing the principle of protein-dye binding. Anal. Biochem. 72:248 254.

Dhindsa, R.S., P. Plumb-Dhindsa, and T.A. Thorpe. 1981. Leaf senescence: Correlated with increased leaves of membrane permeability and lipid peroxidation and decreased levels of superoxide dismutase and catalase. J. Expt. Bot. 32:93-101.

Gautam, P., B. Lal, R. Raja, M.J. Baig, D. Haldar, L. Rath, M. Shahid, R. Tripathi, S. Mohanty, P. Bhattacharyya, and A.K. Nayak. 2014a Post-flood nitrogen and basal phosphorus management affects survival, metabolic changes and anti-oxidant enzyme activities of submerged rice (Oryza sativa). Funct. Plant Biol. 41:1284-1294.

Gautam, P., B. Lal, R. Tripathi, M. Shahid, M.J. Baig, R. Raja, S. Maharana, and A.K. Nayak. 2016. Role of silica and nitrogen interaction in submergence tolerance of rice. Environ. Expt. Bot. 125:98-109.

Gautam, P., A.K. Nayak, B. Lal, P. Bhattacharyya, R. Tripathi, N. Shahid, S. Mohanty, R. Raja, and B.B. Panda. 2014b. Submergence tolerance in relation to application time of nitrogen and phosphorus in rice (Oryza sativa L.). Environ. Exp. Bot. 99:159-166.

Habibzadeh, F., A. Sorooshzadeh, H. Pirdashti, and S.A.M. Modarres-Sanavy. 2013. Alleviation of waterlogging damage by foliar application of nitrogen compounds and tricyclazole in canola. Austral. J. Crop Sci. 7:401-406.

Heath, R.L. and L. Packer. 1968. Photoperoxidation in isolated chloroplasts. I. Kinetics and stoichiometry of fatty acid peroxidation. Arch. Biochem. Biophys. 125:189-198.

Huynh, L.N., T. VanToai, J. Streeter, and G. Banowetz. 2005. Regulation of flooding tolerance of $S A G 12$ : ipt Arabidopsis plants by cytokinin. J. Expt. Bot. 56:1397-1407.

Jiang, Y. and K. Wang. 2006. Growth, physiological and anatomical responses of creeping bentgrass cultivars to different depths of waterlogging. Crop Sci. 46:2420-2426.

Kawano, N., E. Ella, O. Ito, Y. Yamauchi, and K. Tanak. 2002. Metabolic changes in rice seedlings with different submergence tolerance after desubmergence. Environ. Expt. Bot. 47:195-203.

Koehler, L.H. 1952. Differentiation of carbohydrates by anthrone reaction rate and color intensity. Anal. Chem. 24:1576-1579.
Lal, B., P. Gautam, L. Rath, D. Haldar, B.B. Panda, R. Raja, M. Shahid, R. Tripathi, P. Bhattacharyya, S. Mohanty, and A.K. Nayak. 2015. Effect of nutrient application on growth, metabolic and enzymatic activities of rice seedlings during flooding stress and subsequent re-aeration. J. Agron. Crop Sci. 201:138-151.

Liu, M. and Y. Jiang. 2015. Genotypic variation in growth and metabolic responses of perennial ryegrass exposed to short-term waterlogging and submergence stress. Plant Physiol. Biochem. 95:57-64.

Luo, F., K.A. Nagel, H. Scharr, B. Zeng, U. Schurr, and S. Matsubara. 2011. Recovery dynamics of growth, photosynthesis and carbohydrate accumulation after de-submergence: A comparison between two wetland plants showing escape and quiescence strategies. Ann. Bot. (Lond.) 107:49-63.

National Turfgrass Evaluation Program (NTEP). 2016. National Turfgrass Evaluation Program. 1 July 2016. <http://www.ntep.org>.

O'Brien, J.A. and E. Benkova. 2013. Cytokinin cross-talking during biotic and abiotic stress responses. Front. Plant Sci. 4:451.

Panda, D. and R.K. Sarkar. 2013. Characterization of leaf gas exchange and anti-oxidant defense of rice (Oryza sativa L.) cultivars differing in submergence tolerance owing to complete submergence and consequent re-aeration. Agr. Res. 2:301-308.

Panda, D., S.G. Sharma, and R.K. Sarkar. 2008. Chlorophyll fluorescence parameters, $\mathrm{CO}_{2}$ photosynthetic rate and regeneration capacity as a result of complete submergence and subsequent re-emergence in rice (Oryza sativa L.). Aquat. Bot. 88:127-133.

Radha, J., S.P. Singh, A. Singh, S. Singh, A. Chandra, and S. Solomon. 2015. Response of foliar application of nitrogen compounds on sugarcane grown under waterlogging stress. Sugar Tech. 18:443-436.

Ren, B., Y. Zhu, J. Zhang, S. Dong, P. Liu, and B. Zhao. 2016. Effects of spraying exogenous hormone 6-benzyladenine (6-BA) after waterlogging on grain yield and growth of summer maize. Field Crops Res. 188:96104.

SAS Procedures Guide. 2004. SAS Institute Inc., Release 9.1 Edition, Cary, NC.

Tan, S., M. Zhu, and Q. Zhang. 2010. Physiological responses of bermudagrass (Cynodon dactylon) to submergence. Acta Physiol. Plant. 32:133140 .

Tereshonok, D.V., A.Y. Stepanov, Y.I. Dolgikha, E.S. Osipova, D.V. Belyaev, G.R. Kudoyarova, L.B. Vysotskaya, and B.B. Vartapetian. 2011. Effect of the ipt gene expression on wheat tolerance to root flooding. Russ. J. Plant Physiol. 58:799-807.

Yu, X., N. Luo, J. Yan, J. Tang, S. Liu, and Y. Jiang. 2012. Differential growth response and carbohydrate metabolism of global collection of perennial ryegrass accessions to submergence and recovery following de-submergence. J. Plant Physiol. 169:1040-1049.

Zhang, J. and M.B. Kirkham. 1996. Antioxidant responses to drought in sunflower and sorghum seedlings. New Phytol. 132:361-373.

Zwack, P.J. and A.M. Rashotte. 2015. Interactions between cytokinin signalling and abiotic stress responses. J. Expt. Bot. 66:48634871. 